\title{
A general form of an alternative functional equation related to the Jensen's functional equation
}

\author{
Nataphan Kitisin ${ }^{\mathrm{a}, *}$, Choodech Srisawat ${ }^{\mathrm{b}}$
}

a Department of Mathematics and Computer Science, Faculty of Science, Chulalongkorn University, Bangkok 10330 Thailand

b Department of Mathematics, Faculty of Science, Udon Thani Rajabhat University, Udon Thani 41000 Thailand

*Corresponding author, e-mail: nataphan.k@chula.ac.th

ABSTRACT: Given integers $\alpha, \beta, \gamma$ such that $(\alpha, \beta, \gamma) \neq(k,-2 k, k)$ for all $k \in \mathbb{Z}$, we will establish a criterion for the existence of the general solution of the alternative Jensen's functional equation of the form $f\left(x y^{-1}\right)-2 f(x)+f(x y)=$ 0 or $\alpha f\left(x y^{-1}\right)+\beta f(x)+\gamma f(x y)=0$, where $f$ is a mapping from a group $(G, \cdot)$ to a uniquely divisible abelian group $(H,+)$. We also find the general solution in the case when $G$ is a cyclic group.

KEYWORDS: alternative equation, Jensen's functional equation, additive function

MSC2010: 39B52 39B05

\section{INTRODUCTION}

The alternative functional equations related to the Cauchy functional equation

$$
f(x+y)=f(x)+f(y)
$$

has been widely investigated. For instance, Kannappan et al [1] investigated the solutions of the alternative Cauchy functional equation

$$
\begin{aligned}
& (f(x+y)-a f(x)-b f(y)) \\
& \quad(f(x+y)-f(x)-f(y))=0 .
\end{aligned}
$$

Ger [2] extended the results in [1] to the alternative functional equation

$$
\begin{aligned}
& (f(x+y)-a f(x)-b f(y)) \\
& \quad(f(x+y)-c f(x)-d f(y))=0 .
\end{aligned}
$$

Kuczma [3] found that in the case when $a=b=-1$, the alternative Cauchy functional equation (2) on a semigroup is actually equivalent to the Cauchy functional equation (1). Forti [4] studied the general solution of (2) in a more general setting of the form

$$
\begin{gathered}
(c f(x+y)-a f(x)-b f(y)-d) \\
\quad(f(x+y)-f(x)-f(y))=0,
\end{gathered}
$$

by extending the work of Kannappan et al [1].
Nakmahachalasint [5] has studied the alternative Jensen's functional equation of the form

$$
f(x) \pm 2 f(x y)+f(x y 2)=0
$$

on a semigroup.

Srisawat et al [6] gave a criterion for the existence of the general solution for the functional equation

$$
\begin{aligned}
& f\left(x y^{-1}\right)-2 f(x)+f(x y)=0 \quad \text { or } \\
& f\left(x y^{-1}\right)-\lambda f(x)+f(x y)=0,
\end{aligned}
$$

where $f$ is a mapping from a group to a uniquely divisible abelian group. One of the results in [6] said that, for $\lambda \notin\{0,-1,-2\}$, the above alternative functional equation is equivalent to the Jensen's functional equation

$$
f(x)-2 f(x y)+f\left(x y^{2}\right)=0
$$

for all $x, y$ in the domain, which has significantly extended many other work on (3) (see Le et al [7], $\mathrm{Ng}[8,9]$, and Stetkær [10]).

In this paper, we studied the alternative Jensen's functional equation in a more general setting. In other words, given integers $\alpha, \beta, \gamma$ with

$$
(\alpha, \beta, \gamma) \neq(k,-2 k, k) \text { for all } k \in \mathbb{Z},
$$

we will find a criterion of the existence of the general solution of the alternative Jensen's functional 
equation of the form

$$
\begin{aligned}
& f\left(x y^{-1}\right)-2 f(x)+f(x y)=0 \quad \text { or } \\
& \alpha f\left(x y^{-1}\right)+\beta f(x)+\gamma f(x y)=0
\end{aligned}
$$

when $f$ is a mapping from a group $(G, \cdot)$ to a uniquely divisible abelian group $(H,+)$. Then we show that, if $\beta \neq \alpha+\gamma$ and $(\beta, \gamma) \notin\{(0, \alpha),(\alpha, \alpha)\}$, then the above alternative functional equation is equivalent to the Jensen's functional equation (3), in the sense that their sets of solution are the same. Furthermore, we also find the general solution in the case when the domain $G$ is a cyclic group.

\section{NOTATIONS AND DEFINITIONS}

Throughout the paper, we will use the following notations. Let $(G, \cdot)$ be a group and $(H,+)$ be a uniquely divisible abelian group. Note that $(H,+)$ is uniquely divisible if and only if it has two following properties.

(i) For all $a \in H$ and $n \in \mathbb{Z}^{+}$, there exists $b \in H$ such that $n b=a$.

(ii) For all $a, b \in H$ and $n \in \mathbb{Z}^{+}$, if $n a=n b$, then $a=b$.

Next, we will introduce the notations for sequences $\left(a_{k}\right)_{k \in \mathbb{Z}}$ in $H$ as follows.

Notation 1 We denote $\left(a_{k}\right)_{k \in \mathbb{Z}}=(\bar{\alpha}, \bar{\beta})$ when there exists $k_{0} \in \mathbb{Z}$ with $a_{i}=\alpha$ for all $i<k_{0}$ and $a_{i}=\beta$ for all $i \geqslant k_{0}$, i.e.,

$$
(\ldots, \alpha, \alpha, \beta, \beta, \ldots)=(\bar{\alpha}, \bar{\beta}) .
$$

Notation 2 We denote $\left(a_{k}\right)_{k \in \mathbb{Z}}=(\bar{\alpha}, \beta, \bar{\gamma})$ when there exists $k_{0} \in \mathbb{Z}$ with $a_{i}=\alpha$ for all $i<k_{0}, a_{k_{0}}=\beta$, and $a_{i}=\gamma$ for all $i>k_{0}$, i.e.,

$$
(\ldots, \alpha, \alpha, \beta, \gamma, \gamma, \ldots)=(\bar{\alpha}, \beta, \bar{\gamma}) .
$$

Notation 3 Let $p$ be a positive integer. We denote $\left(a_{k}\right)_{k \in \mathbb{Z}}=\left(\overline{\alpha_{0}, \ldots, \alpha_{p-1}}\right)$ when there exists $k_{0} \in \mathbb{Z}$ such that $a_{i}=\alpha_{k_{0}+i(\bmod p)}$ for all $i \in \mathbb{Z}$. In other words, $\left(\overline{\alpha_{0}, \ldots, \alpha_{p-1}}\right)$ is a periodic sequence of period p, i.e.,

$\left(\ldots, \alpha_{0}, \ldots, \alpha_{p-1}, \alpha_{0}, \ldots, \alpha_{p-1}, \ldots\right)=\left(\overline{\alpha_{0}, \ldots, \alpha_{p-1}}\right)$.

Given integers $\alpha, \beta, \gamma$ as in (4) and a function $f: G \rightarrow H$. For every pair of $x, y \in G$, we will define

$$
\mathscr{F}_{y}^{(\alpha, \beta, \gamma)}(x):=\alpha f\left(x y^{-1}\right)+\beta f(x)+\gamma f(x y),
$$

and

$$
J_{y}(x):=f\left(x y^{-1}\right)-2 f(x)+f(x y) .
$$

In addition, we denote the statement

$$
\mathscr{P} f_{y}^{(\alpha, \beta, \gamma)}(x):=\left(J_{y}(x)=0 \quad \text { or } \quad \mathscr{F}_{y}^{(\alpha, \beta, \gamma)}(x)=0\right) .
$$

The set of solution to the statement $\mathscr{P} f_{y}^{(\alpha, \beta, \gamma)}(x)$ will be denoted by $\mathscr{A}_{(G, H)}^{(\alpha, \beta, \gamma)}$, i.e.,

$$
\mathscr{A}_{(G, H)}^{(\alpha, \beta, \gamma)}:=\left\{f: G \rightarrow H \mid \mathscr{P} f_{y}^{(\alpha, \beta, \gamma)}(x), \forall x, y \in G\right\},
$$

while the set of solution of $J_{y}(x)=0$ is denoted by

$$
\mathscr{J}_{(G, H)}:=\left\{f: G \rightarrow H \mid J_{y}(x)=0, \quad \forall x, y \in G\right\} .
$$

For the sake of convenience, we will refer the conditions of integers $\alpha, \beta$ and $\gamma$ as in the following equation,

$$
\left.\begin{array}{ll} 
& \beta=\alpha+\gamma \\
\text { or } & \beta=0 \text { and } \gamma=\alpha \\
\text { or } & (\beta, \gamma)=(\alpha, \alpha) .
\end{array}\right\}
$$

\section{AUXILIARY LEMMAS}

Lemma 1 Let $f \in \mathscr{A}_{(G, H)}^{(\alpha, \beta, \gamma)}$ and $x, y \in G$. If $J_{y}(x) \neq$ 0 , then $\alpha=\gamma$ or $f\left(x y^{-1}\right)=f(x y)$.

Proof: Assume that $J_{y}(x) \neq 0$. By the alternative in $\mathscr{P} f_{y}^{(\alpha, \beta, \gamma)}(x)$ and $\mathscr{P} f_{y^{-1}}^{(\alpha, \beta, \gamma)}(x)$, we get $\mathscr{F}_{y}^{(\alpha, \beta, \gamma)}(x)=$ 0 and $\mathscr{F}_{y^{-1}}^{(\alpha, \beta, \gamma)}(x)=0$, respectively. Therefore, $\mathscr{F}_{y}^{(\alpha, \beta, \gamma)}(x)-\mathscr{F}_{y^{-1}}^{(\alpha, \beta, \gamma)}(x)=0$, i.e.,

$$
(\alpha-\gamma)\left(f\left(x y^{-1}\right)-f(x y)\right)=0 .
$$

Hence $\alpha=\gamma$ or $f\left(x y^{-1}\right)=f(x y)$ as desired.

In the following lemma, we will give a necessary condition for a function $f \in \mathscr{A}_{(G, H)}^{(0, \beta, 0)}$.

Lemma 2 If $f \in \mathscr{A}_{(G, H)}^{(0, \beta, 0)}$ and $x, y \in G$, then $J_{y}(x)=0$.

Proof: Let $f \in \mathscr{A}_{(G, H)}^{(0, \beta, 0)}$ and $x, y \in G$. Suppose $J_{y}(x) \neq 0$. If $\beta=0$, then it is a contradiction to (4). Hence we must have $\beta \neq 0$. From $\mathscr{F}_{y}^{(0, \beta, 0)}(x)=0$, we get $f(x)=0$. Next, we will consider the alternative in $\mathscr{P} f_{y}^{(0, \beta, 0)}\left(x y^{-1}\right)$ as follows.

Case (i). Assume that $\mathscr{F}_{y}^{(0, \beta, 0)}\left(x y^{-1}\right)=0$. We have $f\left(x y^{-1}\right)=0$. By the alternative in $\mathscr{P} f_{y}^{(0, \beta, 0)}(x y)$, we obtain that

$$
f\left(x y^{2}\right)-2 f(x y)=0 \text { or } \beta f(x y)=0 .
$$

By the alternative in $\mathscr{P} f_{y}^{(0, \beta, 0)}\left(x y^{2}\right)$, we get

$f(x y)-2 f\left(x y^{2}\right)+f\left(x y^{3}\right)=0$ or $\beta f\left(x y^{2}\right)=0$. 
Combining (7) and (8), we conclude that

$$
f\left(x y^{3}\right)-3 f(x y)=0 \text { or } f(x y)=0 .
$$

By $f\left(x y^{-1}\right)=0$ and (9), the alternative in $\mathscr{P} f_{y^{2}}^{(0, \beta, 0)}(x y)$ gives $f(x y)=0$. By calculation, we get $J_{y}(x)=0$, a contradiction to the fact that $J_{y}(x) \neq 0$.

Case (ii). Assume that $J_{y}\left(x y^{-1}\right)=0$, i.e.,

$$
f\left(x y^{-2}\right)-2 f\left(x y^{-1}\right)=0 .
$$

By (10) and the alternative in $\mathscr{P} f_{y}^{(0, \beta, 0)}\left(x y^{-2}\right)$, we have

$$
f\left(x y^{-3}\right)-3 f\left(x y^{-1}\right)=0 \text { or } f\left(x y^{-1}\right)=0 .
$$

By (11) and the alternative in $\mathscr{P} f_{y^{2}}^{(0, \beta, 0)}\left(x y^{-1}\right)$, we get

$$
f\left(x y^{-1}\right)+f(x y)=0 \text { or } f\left(x y^{-1}\right)=0 .
$$

If $f\left(x y^{-1}\right)+f(x y)=0$, then we obtain $J_{y}(x)=0$, a contradiction. Hence we have $f\left(x y^{-1}\right)=0$. By a similar argument in (i), we get a contradiction.

Corollary 1 Let $f \in \mathscr{A}_{(G, H)}^{(\alpha, \beta, \alpha)}$ and $x, y \in G$. If $J_{y}(x) \neq 0$, then $\alpha \neq 0$.

Proof: The proof is completed by Lemma 2 .

Lemma 3 Let $f \in \mathscr{A}_{(G, H)}^{(\alpha, \beta, \gamma)}$ with $\alpha \neq \gamma$ and $x, y \in G$. (i) If $J_{y}\left(x y^{-1}\right) \neq 0$ and $J_{y}(x) \neq 0$, then $J_{y}(x y) \neq 0$. (ii) If $J_{y}\left(x y^{-1}\right)=0$ and $J_{y}(x)=0$, then $J_{y}(x y)=0$.

Proof: We will prove each property by contradiction as follows.

(i) Assume that $J_{y}\left(x y^{-1}\right) \neq 0, J_{y}(x) \neq 0$ but $J_{y}(x y)=0$. From $J_{y}\left(x y^{-1}\right) \neq 0$ and $J_{y}(x) \neq 0$, Lemma 1 gives

$$
f\left(x y^{-2}\right)=f(x) \text { and } f\left(x y^{-1}\right)=f(x y) .
$$

Eliminating $f(x y)$ from $J_{y}(x y)=0$ and (12), we get

$$
2 f\left(x y^{-1}\right)-f(x)-f\left(x y^{2}\right)=0 .
$$

We will consider the alternative in $\mathscr{P} f_{y^{2}}^{(\alpha, \beta, \gamma)}(x)$.

- Suppose $J_{y^{2}}(x) \neq 0$. By Lemma 1 , we have

$$
f(x)=f\left(x y^{2}\right) .
$$

By (12) and (14), we get $J_{y^{2}}(x)=0$, a contradiction.
- Suppose $J_{y^{2}}(x)=0 . \quad$ Eliminating $f\left(x y^{-2}\right)$ and $f\left(x y^{2}\right)$ from (12), (13) and $J_{y^{2}}(x)=0$, we get

$$
f\left(x y^{-1}\right)=f(x) \text {. }
$$

By (12) and (15), we obtain $J_{y}(x)=0$, a contradiction.

(ii) Assume that $J_{y}\left(x y^{-1}\right)=0, J_{y}(x)=0$ but $J_{y}(x y) \neq 0$. Eliminating $f\left(x y^{-1}\right)$ from $J_{y}\left(x y^{-1}\right)=0$ and $J_{y}(x)=0$, we get

$$
f\left(x y^{-2}\right)-3 f(x)+2 f(x y)=0 .
$$

From $J_{y}(x y) \neq 0$, Lemma 1 gives

$$
f(x)=f\left(x y^{2}\right) .
$$

Next, we will consider the alternative in $\mathscr{P} f_{y^{2}}^{(\alpha, \beta, \gamma)}(x)$.

- Suppose $J_{y^{2}}(x) \neq 0$. By Lemma 1 , we have

$$
f\left(x y^{-2}\right)=f\left(x y^{2}\right) .
$$

By (17) and (18), we obtain that $J_{y^{2}}(x)=$ 0 , a contradiction.

- Suppose $J_{y^{2}}(x)=0 . \quad$ Eliminating $f\left(x y^{-2}\right)$ and $f\left(x y^{2}\right)$ from (16), (17) and $J_{y^{2}}(x)=0$, we have

$$
f(x)=f(x y) .
$$

By (17) and (19), we get $J_{y}(x y)=0$, a contradiction.

Corollary 2 Let $f \in \mathscr{A}_{(G, H)}^{(\alpha, \beta, \gamma)}$ with $\alpha \neq \gamma$ and $x, y \in G$.

(i) If $J_{y}\left(x y^{-1}\right) \neq 0$ and $J_{y}(x)=0$, then $J_{y}(x y) \neq 0$.

(ii) If $J_{y}\left(x y^{-1}\right)=0$ and $J_{y}(x) \neq 0$, then $J_{y}(x y)=0$.

Proof: It should be noted that $J_{y^{-1}}(x)=J_{y}(x)$ and $J_{y^{-1}}\left(x y^{-1}\right)=J_{y}\left(x y^{-1}\right)$. The proof is completed by replacing $y$ by $y^{-1}$ in Lemma 3 .

Lemma 4 Let $f \in \mathscr{A}_{(G, H)}^{(\alpha, \beta, \gamma)}$ with $\alpha \neq \gamma$ and $x, y \in G$. (i) If $J_{y}\left(x y^{-1}\right) \neq 0$ and $J_{y}(x) \neq 0$, then $\beta=\alpha+\gamma$.

(ii) If $J_{y}\left(x y^{-1}\right)=0$ and $J_{y}(x) \neq 0$, then $(\beta, \gamma)=$ $(0,-\alpha)$.

Proof: We will prove each property by contradiction as follows. 
(i) Assume that $J_{y}\left(x y^{-1}\right) \neq 0, J_{y}(x) \neq 0$ and $\beta \neq$ $\alpha+\gamma$. By Lemma 1, we obtain that

$$
f\left(x y^{-2}\right)=f(x) \text { and } f\left(x y^{-1}\right)=f(x y) .
$$

From $J_{y}\left(x y^{-1}\right) \neq 0$ and $J_{y}(x) \neq 0$, the alternative in $\mathscr{P} f_{y}^{(\alpha, \beta, \gamma)}\left(x y^{-1}\right)$ and $\mathscr{P} f_{y}^{(\alpha, \beta, \gamma)}(x)$ gives

$$
\mathscr{F}_{y}^{(\alpha, \beta, \gamma)}\left(x y^{-1}\right)=0 \text { and } \mathscr{F}_{y}^{(\alpha, \beta, \gamma)}(x)=0 \text {, }
$$

respectively. By (20) and (21), we get

$$
\begin{aligned}
& (\alpha+\gamma) f(x)+\beta f(x y)=0 \quad \text { and } \\
& \beta f(x)+(\alpha+\gamma) f(x y)=0 .
\end{aligned}
$$

Eliminating $f(x y)$ from (22), we have

$$
(\beta-\alpha-\gamma)(\beta+\alpha+\gamma) f(x)=0 .
$$

From $\beta \neq \alpha+\gamma$, we conclude that $f(x)=0$ or $\beta=-\alpha-\gamma$.

- Suppose $f(x)=0$. By (22), we have

$$
\beta f(x y)=0 \text { and }(\alpha+\gamma) f(x y)=0 .
$$

If $f(x y)=0$, then $f\left(x y^{-1}\right)=0$ by (20). Hence we calculate $J_{y}(x)=0$, a contradiction to the fact that $J_{y}(x) \neq 0$. Thus we get $\alpha+\gamma=0$ and $\beta=0$, a contradiction to the fact that $\beta \neq \alpha+\gamma$.

- Suppose $\beta=-\alpha-\gamma$. Substituting $\beta=$ $-\alpha-\gamma$ in (22), we obtain that

$$
(\alpha+\gamma)(f(x)-f(x y))=0 .
$$

If $\alpha+\gamma=0$, then $\beta=0$ which contra$\operatorname{dicts} \beta \neq \alpha+\gamma$. Thus we must have $f(x)=f(x y)$. Since $f\left(x y^{-1}\right)=f(x y)$ in (20), we get $J_{y}(x)=0$, a contradiction.

Hence $\beta=\alpha+\gamma$.

(ii) Assume that $J_{y}\left(x y^{-1}\right)=0$ and $J_{y}(x) \neq 0$. From $J_{y}(x) \neq 0$, by Lemma 1 , we have

$$
f\left(x y^{-1}\right)=f(x y) .
$$

By $J_{y}(x) \neq 0$ again, the alternative in $\mathscr{P} f_{y}^{(\alpha, \beta, \gamma)}(x)$ gives $\mathscr{F}_{y}^{(\alpha, \beta, \gamma)}(x)=0$. Substituting $f\left(x y^{-1}\right)$ from (23) in $\mathscr{F}_{y}^{(\alpha, \beta, \gamma)}(x)=0$, we obtain that

$$
\beta f(x)+(\alpha+\gamma) f(x y)=0 .
$$

On the other hand, we substitute $f\left(x y^{-1}\right)$ from (23) in $J_{y}\left(x y^{-1}\right)=0$ to get

$$
f\left(x y^{-2}\right)-2 f(x y)+f(x)=0 .
$$

Since $J_{y}\left(x y^{-1}\right)=0$ and $J_{y}(x) \neq 0$, Corollary 2 give $J_{y}\left(x y^{-2}\right) \neq 0$. By Lemma 1 and (23), we have

$$
f\left(x y^{-3}\right)=f(x y) .
$$

From $J_{y}\left(x y^{-2}\right) \neq 0$, the alternative in $\mathscr{P} f_{y}^{(\alpha, \beta, \gamma)}\left(x y^{-2}\right)$ gives $\mathscr{F}_{y}^{(\alpha, \beta, \gamma)}\left(x y^{-2}\right)=0$. By (23), (26) and $\mathscr{F}_{y}^{(\alpha, \beta, \gamma)}\left(x y^{-2}\right)=0$, we get

$$
\beta f\left(x y^{-2}\right)+(\alpha+\gamma) f(x y)=0 .
$$

Eliminating $f\left(x y^{-2}\right)$ from (25) and (27), we have

$$
\beta f(x)-(\alpha+2 \beta+\gamma) f(x y)=0 .
$$

By (24), (28), and simplifying, we obtain that

$$
\beta(f(x)-f(x y))=0 .
$$

If $f(x)-f(x y)=0$, then by (23), we have $J_{y}(x)=0$, a contradiction. Hence we must get $\beta=0$. Thus (28) reduces to

$$
(\alpha+\gamma) f(x y)=0 .
$$

Suppose in a contrary that $\alpha+\gamma \neq 0$. Thus $f(x y)=0$ and so is $f\left(x y^{-1}\right)$ by (23). By (25), we obtain that

$$
f\left(x y^{-2}\right)+f(x)=0 .
$$

From $J_{y}\left(x y^{-1}\right)=0$ and $J_{y}(x) \neq 0$, Corollary 2 give $J_{y}(x y)=0$, i.e.,

$$
f(x)+f\left(x y^{2}\right)=0 .
$$

Thus by (30), (31) and $\alpha+\gamma \neq 0$, the alternative in $\mathscr{P} f_{y^{2}}^{(\alpha, 0, \gamma)}(x)$ give $f(x)=0$. Then $J_{y}(x)=0$, a contradiction. Therefore, we must get $\alpha+\gamma=0$ and so $(\beta, \gamma)=(0,-\alpha)$.

\section{MAIN RESULTS AND EXAMPLES}

Now we state the following theorem in the work of Srisawat et al [6], which will be useful in proving our results.

Theorem 1 If $f \in \mathscr{A}_{(G, H)}^{(1, \beta, 1)} \backslash \mathscr{J}_{(G, H)}$, then $\beta \in\{0,1,2\}$. Moreover, if $x, y \in G$, then one of the following properties must hold:

(i) $\beta=0$ and $\left(f\left(x y^{n}\right)\right)_{n \in \mathbb{Z}}=(\overline{-a}, \bar{a})$ for some $a \in H \backslash\{0\}$.

(ii) $\beta=1$ and

(a) $\left(f\left(x y^{n}\right)\right)_{n \in \mathbb{Z}}=(\overline{a, b,-a-b})$ for some $a, b \in H$ with $(a, b) \neq(0,0)$, or 
(b) $\left(f\left(x y^{n}\right)\right)_{n \in \mathbb{Z}}=(\bar{a},-2 a, \bar{a})$ for some $a \in H \backslash\{0\}$, or

(c) $\left(f\left(x y^{n}\right)\right)_{n \in \mathbb{Z}}=(\overline{-2 a, a, \ldots, a})$, a periodic sequence of odd period $p \geqslant 5$, for some $a \in$ $H \backslash\{0\}$.

(iii) $\beta=2$ and $f\left(x y^{n}\right)=(-1)^{n}(f(x)-n(f(x)+$ $f(x y)))$ for all $n \in \mathbb{Z}$.

We will first provide the following two lemmas which will eventually be used in our main theorem.

Lemma 5 Let $f \in \mathscr{A}_{(G, H)}^{(\alpha, \beta, \alpha)} \backslash \mathscr{J}_{(G, H)}$. If $x, y \in G$, then one of the following properties holds:

(i) $\beta=0$ and $\left(f\left(x y^{n}\right)\right)_{n \in \mathbb{Z}}=(\overline{-a}, \bar{a})$ for some $a \in H$.

(ii) $\beta=\alpha$ and

(a) $\left(f\left(x y^{n}\right)\right)_{n \in \mathbb{Z}}=(\overline{a, b,-a-b})$ for some $a, b \in H$, or

(b) $\left(f\left(x y^{n}\right)\right)_{n \in \mathbb{Z}}=(\bar{a},-2 a, \bar{a})$ for some $a \in H$, or

(c) $\left(f\left(x y^{n}\right)\right)_{n \in \mathbb{Z}}=(\overline{-2 a, a, \ldots, a})$, a periodic sequence of odd period $p \geqslant 5$, for some $a \in H$.

(iii) $\beta=2 \alpha$ and $f\left(x y^{n}\right)=(-1)^{n}(f(x)-n(f(x)+$ $f(x y))$ ) for all $n \in \mathbb{Z}$.

Proof: Assume that the assumption in the lemma holds. From Corollary 1, we must have $\alpha \neq 0$. By direct substitution, we get $\mathscr{A}_{(G, H)}^{(\alpha, \beta, \alpha)} \backslash \mathscr{J}_{(G, H)}=$ $\mathscr{A}_{(G, H)}^{(1, \beta / \alpha, 1)} \backslash \mathscr{J}_{(G, H)}$. Thus Theorem 1 gives $\beta / \alpha \in$ $\{0,1,2\}$, and $f$ satisfies one of the properties in the lemma.

Lemma 6 Let $f \in \mathscr{A}_{(G, H)}^{(\alpha, \beta, \gamma)} \backslash \mathscr{J}_{(G, H)}$ with $\alpha \neq \gamma$. Moreover, if $x, y \in G$, then one of the following properties holds:

(i) $\beta=\alpha+\gamma$ and $f\left(x y^{n}\right)=(-1)^{n} a$ for all $n \in \mathbb{Z}$ and for some $a \in H$, or

(ii) $(\beta, \gamma)=(0,-\alpha)$ and

(a) $\left(f\left(x y^{n}\right)\right)_{n \in \mathbb{Z}}=(\overline{a, b})$ for some $a, b \in H$, or

(b) $\left(f\left(x y^{n}\right)\right)_{n \in \mathbb{Z}}=(\overline{2 a-b, a, b, a})$ for some $a, b \in H$.

(iii) $f\left(x y^{n}\right)=f(x)+n(f(x y)-f(x))$ for all $n \in \mathbb{Z}$.

Proof: Assume that the assumption in the lemma holds. By the definition of $\mathscr{A}_{(G, H)}^{(\alpha, \beta, \gamma)}$ and $\mathscr{J}_{(G, H)}$, one of the following properties holds:

(i) $J_{y}\left(x y^{n}\right) \neq 0$ for all $n \in \mathbb{Z}$.

(ii) There exists $m \in \mathbb{Z}$ such that

(a) $J_{y}\left(x y^{m}\right) \neq 0$ and $J_{y}\left(x y^{m-1}\right)=0$, or

(b) $J_{y}\left(x y^{m}\right) \neq 0$ and $J_{y}\left(x y^{m+1}\right)=0$.

(iii) $J_{y}\left(x y^{n}\right)=0$ for all $n \in \mathbb{Z}$.

Case (i). Assume that $J_{y}\left(x y^{n}\right) \neq 0$ for all $n \in \mathbb{Z}$. Lemma 4 gives $\beta=\alpha+\gamma$. By Lemma 1 , we get

$$
f\left(x y^{n-1}\right)=f\left(x y^{n+1}\right) \text { for all } n \in \mathbb{Z} \text {. }
$$

From $J_{y}(x) \neq 0$, the alternative in $\mathscr{P} f_{y}^{(\alpha, \alpha+\gamma, \gamma)}(x)$ gives $\mathscr{F}_{y}^{(\alpha, \alpha+\gamma, \gamma)}(x)=0$, i.e.,

$$
\alpha f\left(x y^{-1}\right)+(\alpha+\gamma) f(x)+\gamma f(x y)=0 .
$$

By (32) with $n=0$ and (33), we get

$$
(\alpha+\gamma)(f(x)+f(x y))=0
$$

- Suppose $f(x)+f(x y)=0$. Let $f(x)=a$. We have $f(x y)=-a$. By (32), we conclude that

$$
f\left(x y^{n}\right)= \begin{cases}a & \text { if } n \text { is even } \\ -a & \text { if } n \text { is odd. }\end{cases}
$$

Hence we get $f\left(x y^{n}\right)=(-1)^{n} a$ for all $n \in \mathbb{Z}$.

- Suppose $\alpha+\gamma=0$. That is $\beta=0$. Let $f(x)=a$ and $f(x y)=b$. Therefore, by (32), we obtain that

$$
f\left(x y^{n}\right)= \begin{cases}a & \text { if } n \text { is even } \\ b & \text { if } n \text { is odd }\end{cases}
$$

Thus we have $\left(f\left(x y^{n}\right)\right)_{n \in \mathbb{Z}}=(\overline{a, b})$.

Case (ii). Assume that there exists $m \in \mathbb{Z}$ such that $\left(J_{y}\left(x y^{m}\right) \neq 0\right.$ and $\left.J_{y}\left(x y^{m-1}\right)=0\right)$ or $\left(J_{y}\left(x y^{m}\right) \neq 0\right.$ and $\left.J_{y}\left(x y^{m+1}\right)=0\right)$. Thus Lemma 4 gives $(\beta, \gamma)=(0,-\alpha)$.

- Suppose $J_{y}\left(x y^{m}\right) \neq 0$ and $J_{y}\left(x y^{m-1}\right)=0$. Let $f\left(x y^{m-1}\right)=a$ and $f\left(x y^{m}\right)=b$. From $J_{y}\left(x y^{m}\right) \neq 0$, Lemma 1 gives $f\left(x y^{m-1}\right)=$ $f\left(x y^{m+1}\right)$, i.e., $f\left(x y^{m+1}\right)=a$. From $J_{y}\left(x y^{m-1}\right)=0$, we get $f\left(x y^{m-2}\right)=2 a-b$. Now we have

$$
\begin{gathered}
\left(f\left(x y^{m-2}\right), f\left(x y^{m-1}\right), f\left(x y^{m}\right), f\left(x y^{m+1}\right)\right) \\
=(2 a-b, a, b, a) .
\end{gathered}
$$

From $J_{y}\left(x y^{m-1}\right)=0$ and $J_{y}\left(x y^{m}\right) \neq 0$, Corollary 2 gives $J_{y}\left(x y^{m+1}\right)=0$, i.e.,

$$
f\left(x y^{m}\right)-2 f\left(x y^{m+1}\right)+f\left(x y^{m+2}\right)=0 .
$$

By (35) and (36), we obtain that $f\left(x y^{m+2}\right)=2 a-b$. Since $J_{y}\left(x y^{m}\right) \neq 0$ and $J_{y}\left(x y^{m+1}\right)=0$, Corollary 2 gives $J_{y}\left(x y^{m+2}\right) \neq 0$. By Lemma 1, we get $f\left(x y^{m+3}\right)=a$. From $J_{y}\left(x y^{m+1}\right)=0$ and $J_{y}\left(x y^{m+2}\right) \neq 0$, Corollary 2 gives $J_{y}\left(x y^{m+3}\right)=0$ and so $f\left(x y^{m+4}\right)=b$. As $J_{y}\left(x y^{m+2}\right) \neq 0$ and $J_{y}\left(x y^{m+3}\right)=0$, we have 
$J_{y}\left(x y^{m+4}\right) \neq 0$ by Corollary 2 . Lemma 1 gives $f\left(x y^{m+5}\right)=a$. Thus we obtain that

$$
\begin{gathered}
\left(f\left(x y^{m+2}\right), f\left(x y^{m+3}\right), f\left(x y^{m+4}\right), f\left(x y^{m+5}\right)\right) \\
=(2 a-b, a, b, a) .
\end{gathered}
$$

Similarly, by repeating the process of (37), we get

$$
\begin{gathered}
\left(f\left(x y^{m+6}\right), f\left(x y^{m+7}\right), f\left(x y^{m+8}\right), f\left(x y^{m+9}\right)\right) \\
=(2 a-b, a, b, a)
\end{gathered}
$$

and so on. Eventually, we arrive that

$$
\begin{aligned}
& \left(f\left(x y^{m-2+4 i}\right), f\left(x y^{m-1+4 i}\right), f\left(x y^{m+4 i}\right),\right. \\
& \left.f\left(x y^{m+1+4 i}\right)\right)=(2 a-b, a, b, a)
\end{aligned}
$$

for all $i \geqslant 0$. Moreover, we can similarly repeat the process of (38) for each $f\left(x y^{k}\right)$ with $k \leqslant m-3$ to get $\left(f\left(x y^{n}\right)\right)_{n \in \mathbb{Z}}=$ $(\overline{2 a-b, a, b, a})$.

- If $J_{y}\left(x y^{m}\right) \neq 0$ and $J_{y}\left(x y^{m+1}\right)=0$, then we have the similar results by replacing $x$ by $x y^{2 m}$ and $y$ by $y^{-1}$. Hence

Case (iii). Assume that $J_{y}\left(x y^{n}\right)=0$ for all $n \in \mathbb{Z}$.

$$
f\left(x y^{n+1}\right)-f\left(x y^{n}\right)=f\left(x y^{n}\right)-f\left(x y^{n-1}\right)
$$

for all $n \in \mathbb{Z}$ and we get the the property (iii) as desired.

Now we are ready to prove the main theorem.

Theorem 2 If $f \in \mathscr{A}_{(G, H)}^{(\alpha, \beta, \gamma)} \backslash \mathscr{J}_{(G, H)}$, then (6) holds. Moreover, if $x, y \in G$, then one of the following properties holds:

(i) $\beta=\alpha+\gamma$ and

(a) $f\left(x y^{n}\right)=(-1)^{n} a$ for all $n \in \mathbb{Z}$ and for some $a \in H$, or

(b) $\beta=0$ and

(I) $\left(f\left(x y^{n}\right)\right)_{n \in \mathbb{Z}}=(\overline{a, b})$ for some $a, b \in H$, or

(II) $\left(f\left(x y^{n}\right)\right)_{n \in \mathbb{Z}}=(\overline{2 a-b, a, b, a})$ for some $a, b \in H$, or

(c) $\beta=2 \alpha$ and $f\left(x y^{n}\right)=(-1)^{n}(f(x)-$ $n(f(x)+f(x y)))$ for all $n \in \mathbb{Z}$.

(ii) $(\beta, \gamma)=(0, \alpha)$ and $\left(f\left(x y^{n}\right)\right)_{n \in \mathbb{Z}}=(\overline{-a}, \bar{a})$ for some $a \in H$.

(iii) $(\beta, \gamma)=(\alpha, \alpha)$ and

(a) $\left(f\left(x y^{n}\right)\right)_{n \in \mathbb{Z}}=(\overline{a, b,-a-b})$ for some $a, b \in$ $H$, or

(b) $\left(f\left(x y^{n}\right)\right)_{n \in \mathbb{Z}}=(\bar{a},-2 a, \bar{a})$ for some $a \in H$, or (c) $\left(f\left(x y^{n}\right)\right)_{n \in \mathbb{Z}}=(\overline{-2 a, a, \ldots, a})$, a periodic sequence of odd period $p \geqslant 5$, for some $a \in H$.

(iv) $f\left(x y^{n}\right)=f(x)+n(f(x y)-f(x))$ for all $n \in \mathbb{Z}$.

Proof: Assume that all assumptions in the theorem hold. We will consider the case of an integer $\alpha$ as follows:

(i) If $\alpha=\gamma$, then Lemma 5 , gives $\beta \in\{0, \alpha, 2 \alpha\}$. Moreover, we get the properties (i-c), (ii) and (iii) corresponding to properties (iii), (i) and (ii) of Lemma 5, respectively.

(ii) If $\alpha \neq \gamma$, then we obtain the properties (i-a), (i-b) and (iv) corresponding to properties (i), (ii) and (iii) of Lemma 6, respectively.

Corollary 3 Let $f \in \mathscr{A}_{(G, H)}^{(\alpha, \beta, \gamma)}$. If (6) does not hold, then $f \in \mathscr{J}_{(G, H)}$.

Proof: If (6) does not hold, then, from Theorem 2, $\mathscr{A}_{(G, H)}^{(\alpha, \beta, \gamma)} \backslash \mathscr{J}_{(G, H)}$ is empty. Hence we have the desired result.

In other words, Corollary 3 states that when (6) does not hold, the alternative Jensen's functional equation (5) is equivalent to the Jensen's functional equation (3) for the class of functions from $(G, \cdot)$ to $(H,+)$. On the other hand, when (6) actually holds, (5) is not necessarily equivalent to (3). We will give the following two examples when (5) is not necessarily equivalent to (3) with $\beta=\alpha+\gamma$ or $(\beta, \gamma)=(0, \alpha)$.

Example 1 Given $a \in H \backslash\{0\}$. Let $f: \mathbb{Z} \rightarrow H$ be a function such that

$$
f(n)=(-1)^{n} a \text { for all } n \in \mathbb{Z} .
$$

Note that

$$
f(0)-2 f(1)+f(2)=4 a .
$$

From $a \neq 0$ and $H$ is uniquely divisible, we get $4 a \neq$ 0 . Thus $f \notin \mathscr{J}_{(\mathbb{Z}, H)}$. Given $n, m \in \mathbb{Z}$. If $m$ is odd, then we observe that $n-m$ and $n+m$ have the same parity whereas $n$ and $n+m$ have the opposite. Therefore,

$$
\alpha f(n-m)+(\alpha+\gamma) f(n)+\gamma f(n+m)=0 .
$$

Otherwise, if $m$ is even, then $n-m, n, n+m$ all have the same parity. Hence

$$
f(n-m)-2 f(n)+f(n+m)=0 .
$$

Therefore, $f \in \mathscr{A}_{(\mathbb{Z}, H)}^{(\alpha, \alpha+\gamma, \gamma)} \backslash \mathscr{J}_{(\mathbb{Z}, H)}$. 
Example 2 Given $a, b \in H$ with $a \neq b$. Let $f: \mathbb{Z} \rightarrow$ $H$ be a function such that

$$
f(n)= \begin{cases}a & \text { if } n \text { is even, } \\ b & \text { if } n \text { is odd. }\end{cases}
$$

Note that

$$
f(0)-2 f(1)+f(2)=2 a-2 b .
$$

Since $a \neq b$ and $H$ is uniquely divisible, we have $2 a-2 b \neq 0$. Thus $f \notin \mathscr{J}_{(\mathbb{Z}, H)}$. If $m$ is odd, then we observe that $n-m$ and $n+m$ have the same parity. Therefore,

$$
\alpha f(n-m)-\alpha f(n+m)=0 .
$$

Otherwise, if $m$ is even, then $n-m, n, n+m$ all have the same parity. Hence

$$
f(n-m)-2 f(n)+f(n+m)=0 .
$$

Thus $f \in \mathscr{A}_{(\mathbb{Z}, H)}^{(\alpha, 0,-\alpha)} \backslash \mathscr{J}_{(\mathbb{Z}, H)}$.

\section{GENERAL SOLUTION ON CYCLIC GROUPS}

In this section, we will give the general solution of the alternative Jensen's functional equation (5) on an infinite cyclic group and a finite cyclic group. They are mainly the applications of Theorem 2 . First, we will find all solutions of an infinite cyclic group as in the following theorem.

Theorem 3 Let $(G, \cdot)$ be an infinite cyclic group with $G=\langle g\rangle . f \in \mathscr{A}_{(G, H)}^{(\alpha, \beta, \gamma)}$ if and only if $f \in \mathscr{J}_{(G, H)}$ or one of the following properties must hold:

(i) $\beta=\alpha+\gamma$ and

(a) $f\left(g^{n}\right)=(-1)^{n} a$ for all $n \in \mathbb{Z}$ and for some $a \in H$, or

(b) $\beta=0$ and

(I) $\left(f\left(g^{n}\right)\right)_{n \in \mathbb{Z}}=(\overline{a, b})$ for some $a, b \in H$, or

(II) $\left(f\left(g^{n}\right)\right)_{n \in \mathbb{Z}}=(\overline{2 a-b, a, b, a})$ for some $a, b \in H$, or

(c) $\beta=2 \alpha$ and $f\left(g^{n}\right)=(-1)^{n}(a+n b)$ for all $n \in \mathbb{Z}$ and for some $a, b \in H$.

(ii) $(\beta, \gamma)=(0, \alpha)$ and $\left(f\left(g^{n}\right)\right)_{n \in \mathbb{Z}}=(\overline{-a}, \bar{a})$ for some $a \in H$.

(iii) $(\beta, \gamma)=(\alpha, \alpha)$ and

(a) $\left(f\left(g^{n}\right)\right)_{n \in \mathbb{Z}}=(\overline{a, b,-a-b})$ for some $a, b \in$ $H$, or

(b) $\left(f\left(g^{n}\right)\right)_{n \in \mathbb{Z}}=(\bar{a},-2 a, \bar{a})$ for some $a \in H$, or

(c) $\left(f\left(g^{n}\right)\right)_{n \in \mathbb{Z}}=(\overline{-2 a, a, \ldots, a})$, a periodic sequence of odd period $p \geqslant 5$, for some $a \in H$.
Proof: Assume that $f \in \mathscr{A}_{(G, H)}^{(\alpha, \beta, \gamma)}$. If $f \notin \mathscr{J}_{(G, H)}$, then setting $x=e$ and $y=g$ in Theorem 2, we get that one of the properties (i), (ii) and (iii) must hold. It is obvious to see that the property (iv) of Theorem 2 is redundant. The converse can be directly verified.

Next, we will give the general solution of a finite cyclic group as in the following theorem.

Theorem 4 Let $(G, \cdot)$ be a finite cyclic group of order $m \geqslant 2$ with $G=\langle g\rangle . f \in \mathscr{A}_{(G, H)}^{(\alpha, \beta, \gamma)}$ if and only if $f \in$ $\mathscr{J}_{(G, H)}$ or one of the following properties must hold:

(i) $\beta=\alpha+\gamma$,

(a) $2 \mid m$ and $f\left(g^{n}\right)=(-1)^{n} a$ for all $n \in \mathbb{Z}$ and for some $a \in H$, or

(b) $\beta=0$,

(I) $2 \mid m$ and $\left(f\left(g^{n}\right)\right)_{n \in \mathbb{Z}}=(\overline{a, b})$ for some $a, b \in H$, or

(II) $4 \mid m$ and $\left(f\left(g^{n}\right)\right)_{n \in \mathbb{Z}}=(\overline{2 a-b, a, b, a})$ for some $a, b \in H$, or

(ii) $(\beta, \gamma)=(\alpha, \alpha)$,

(a) $3 \mid m$ and $\left(f\left(g^{n}\right)\right)_{n \in \mathbb{Z}}=(\overline{a, b,-a-b})$ for some $a, b \in H$, or

(b) $\left(f\left(g^{n}\right)\right)_{n \in \mathbb{Z}}=(\overline{-2 a, a, \ldots, a})$, a periodic sequence of odd period $p \geqslant 5$ with $p \mid m$, for some $a \in H$.

Proof: Given one of the above properties, we can directly verify that $f \in \mathscr{A}_{(G, H)}^{(\alpha, \beta, \gamma)}$. Conversely, assume that $f \in \mathscr{A}_{(G, H)}^{(\alpha, \beta, \gamma)} \backslash \mathscr{J}_{(G, H)}$. By setting $x=e$ and $y=g$ in Theorem 2, we have the possibilities in Theorem 3.

However, all the above possibilities are not admissible. Some cases are redundant and some cases are admissible with some additional conditions. It is obvious to see that the property (iv) of Theorem 2 is redundant.

(i) Assume that $\beta=\alpha+\gamma$.

(a) Suppose that $f\left(g^{n}\right)=(-1)^{n} a$ for all $n \in \mathbb{Z}$, for some $a \in H$ and $m$ is odd. We get

$$
a=f(e) \text { and } f\left(g^{m}\right)=-a \text {. }
$$

Since $g^{m}=e$, therefore $a=0$ and so $f \in$ $\mathscr{J}_{(G, H)}$, a contradiction. Thus $m$ must be even.

(b) Suppose that $\left(f\left(g^{n}\right)\right)_{n \in \mathbb{Z}}=(\overline{a, b})$ for some $a, b \in H$ and $m$ is odd. Without loss of generality, we let $f(e)=a$. Then $f\left(g^{m}\right)=$ $b$. Since $g^{m}=e, a=b$ and thus $f \in \mathscr{J}_{(G, H)}$, a contradiction. Hence $m$ must be even. 
(c) Suppose that $\left(f\left(g^{n}\right)\right)_{n \in \mathbb{Z}}=(\overline{2 a-b, a, b, a})$ for some $a, b \in H$ and $4 \nmid m$. Then there exists $k \in \mathbb{Z}$ such that $f\left(g^{k}\right)=2 a-b$. Since $4 \nmid m, f\left(g^{k+m}\right) \in\{a, b\}$. From $m$ is the order of the group $G$, we have $g^{k}=g^{k+m}$. Hence

$$
2 a-b=a \text { or } 2 a-b=b,
$$

which gives $a=b$. Thus $f \in \mathscr{J}_{(G, H)}$, a contradiction. Therefore, we must get $4 \mid \mathrm{m}$.

(d) Suppose that $f\left(g^{n}\right)=(-1)^{n}(a+n b)$ for all $n \in \mathbb{Z}$ and for some $a, b \in H$. Since $e=g^{m}=$ $g^{2 m}$,

$$
a=(-1)^{m}(a+m b)=(-1)^{2 m}(a+2 m b)
$$

which implies that $b=0$ and $m$ is even.

(ii) Assume that $(\beta, \gamma)=(0, \alpha)$ and $\left(f\left(g^{n}\right)\right)_{n \in \mathbb{Z}}=$ $(\overline{-a}, \bar{a})$ for some $a \in H$. Thus there exists $k \in \mathbb{Z}$ such that

$$
f\left(g^{n}\right)= \begin{cases}-a & \text { if } n<k, \\ a & \text { if } n \geqslant k .\end{cases}
$$

Hence $f\left(g^{k-1}\right)=-a$ and $f\left(g^{k+m-1}\right)=a$. Since $m$ is the order of the group $G$, we have $g^{k-1}=$ $g^{k+m-1}$. Thus we must get $a=0$ and so $f \in$ $\mathscr{J}_{(G, H)}$, a contradiction. Therefore, this case will not occur.

(iii) Assume that $(\beta, \gamma)=(\alpha, \alpha)$.

(a) Suppose that $\left(f\left(g^{n}\right)\right)_{n \in \mathbb{Z}}=(\overline{a, b,-a-b})$ for some $a, b \in H$ and $3 \nmid m$. Then $\{0, m, 2 m\}$ is a complete residue modulo 3 . Therefore

$$
\left\{f(e), f\left(g^{m}\right), f\left(g^{2 m}\right)\right\}=\{a, b,-a-b\} .
$$

Since $m$ is the order of $G$, thus $g^{2 m}=g^{m}=e$. Therefore, $a=b=-a-b$, which gives $a=b=0$ and, in turn, $f \in \mathscr{J}_{(G, H)}$, a contradiction. Hence $3 \mid m$.

(b) $\left(f\left(g^{n}\right)\right)_{n \in \mathbb{Z}}=(\bar{a},-2 a, \bar{a})$ for some $a \in H$. Then there exists $k \in \mathbb{Z}$ such that

$$
f\left(g^{n}\right)= \begin{cases}-2 a & \text { if } n=k \\ a & \text { otherwise }\end{cases}
$$

Hence $f\left(g^{k}\right)=-2 a$ and $f\left(g^{k+m}\right)=a$. Since $m$ is the order of the group $G$, we have $g^{k}=$ $g^{k+m}$. Thus we must have $a=0$ and so $f \in$ $\mathscr{J}_{(G, H)}$, a contradiction. Therefore, this case does not occur.

(c) Suppose that $\left(f\left(g^{n}\right)\right)_{n \in \mathbb{Z}}=(\overline{-2 a, a, \ldots, a})$, a periodic sequence of odd period $p \geqslant 5$, for some $a \in H$ and $p \nmid m$. Thus there is $k \in \mathbb{Z}$ such that $f\left(g^{k}\right)=-2 a$. Since $\left(f\left(g^{n}\right)\right)_{n \in \mathbb{Z}}$ is periodic sequence of period $p$ with $p \nmid m$, we must have $f\left(g^{k+m}\right)=a$. But $m$ is the order of $G$, thus $g^{k+m}=g^{k}$. Therefore, $-2 a=a$, which gives $a=0$, and, in turn, $f \in \mathscr{J}_{(G, H)}$, a contradiction. Hence $p \mid m$.

By all of the above considerations, we are done.

\section{REFERENCES}

1. Kannappan PL, Kuczma M (1974) On a functional equation related to the Cauchy equation. Ann Polon Math 30, 49-55.

2. Ger R (1977) On an alternative functional equation. Aequationes Mathematicae 15, 145-162.

3. Kuczma M (1978) On some alternative functional equations. Aequationes Mathematicae 2, 182-198.

4. Forti GL (1979) La soluzione generale dell'equazione funzionale $\{c f(x+y)-a f(x)-b f(y)-d\}\{f(x+$ $y)-f(x)-f(y)\}=0$. Matematiche (Catania) 34, 219-242.

5. Nakmahachalasint P (2012) An alternative Jensen's functional equation on semigroups. ScienceAsia 38, 408-413.

6. Srisawat C, Kitisin N, Nakmahachalasint P (2015) An alternative functional equation of Jensen type on groups. ScienceAsia 41, 280-288.

7. Le C-T, Thai T-H (2011) Jensen's functional equation on the symmetric group $S_{n}$. Aequationes Mathematicae 82, 269-276.

8. Ng CT (2001) Jensen's functional equation on groups, III. Aequationes Mathematicae 62, 143-159.

9. Ng CT (2005) A Pexider-Jensen functional equation on groups. Aequationes Mathematicae 70, 131-153.

10. Stetkær H (2003) On Jensen's functional equation on groups. Aequationes Mathematicae 66, 100-118. 